\title{
Prótese auditiva ancorada ao osso percutânea: benefícios auditivos
}

\section{Percutaneous bone ancored hearing aid: hearing benefits}

\author{
Brenda Catalani' (D), Tyuana Sandim da Silveira Sassi2 (D), Érika Cristina Bucuvic² (D), \\ Luiz Fernando Manzoni Lourençone ${ }^{3,4}$ (1), Kátia de Freitas Alvarenga ${ }^{5}$ (D), Rubens Vuono de Brito Neto ${ }^{3,4}$ (1)
}

\section{RESUMO}

Objetivo: Descrever os benefícios nos limiares auditivos e no desempenho de reconhecimento de sentenças no silêncio e no ruído em indivíduos com a adaptação unilateral do Sistema Ponto ${ }^{\circledR}$. Métodos: Estudo observacional, retrospectivo, de seguimento longitudinal. A casuística foi composta por fontes de dados secundários de dez indivíduos com perda auditiva condutiva ou mista, que foram submetidos à cirurgia com o Sistema Ponto ${ }^{\circledR}$. Os resultados foram analisados nas seguintes condições: a) pré-cirúrgicas: sem AASI; com AASI por condução aérea ou óssea e com o processador Ponto Pro ${ }^{\circledR}$ acoplado a uma banda elástica; b) pós-cirúrgicas: na ativação e após seis meses de uso. Resultados: Os limiares da audiometria tonal por conduções aérea e óssea mantiveram-se estáveis após a cirurgia, enquanto os limiares auditivos em campo livre e o reconhecimento de fala no silêncio e no ruído foram estatisticamente melhores na ativação e após seis meses de uso do Sistema Ponto ${ }^{\circledR}$. Não houve diferença nos resultados com os indivíduos utilizando o Sistema Ponto ${ }^{\circledR}$ com a banda elástica e após a cirurgia. Conclusão: O Sistema Ponto ${ }^{\circledR}$ propiciou benefício nas habilidades auditivas de detecção em todas as frequências testadas, assim como no reconhecimento de sentenças no silêncio e no ruído.

Palavras-chave: Prótese ossicular; Audiometria da fala; Condução óssea; Audiometria; Auxiliares de audição

\begin{abstract}
Purpose: To describe the benefits in hearing thresholds and sentence recognition performance in silence and noise, in users of the unilateral Ponto ${ }^{\mathbb{B}}$ system. Methods: An observational, retrospective, longitudinal study. The sample consisted of secondary data sources from 10 individuals with conductive or mixed hearing loss who underwent surgery with the Ponto ${ }^{\circledR}$ System. The results were analyzed in the following pre-surgical conditions (without hearing aids; with hearing aids by air or bone conduction; with the Ponto Pro ${ }^{\circledR}$ processor with a soft band) and post-surgical (on activation and after six months of use). Results: The thresholds of pure tone audiometry by air and bone conductions remained stable after surgery, while the auditory thresholds in free field and speech recognition in silence and in noise were statistically better when using the Ponto ${ }^{\circledR}$ system. There was no difference between the results obtained with the individuals using Ponto ${ }^{\circledR}$ with soft band and post-surgically. Conclusion: The Ponto ${ }^{\circledR}$ system provided benefits in hearing detection skills in all tested frequencies, as well as, in recognition of the sentence in silence and noise.
\end{abstract}

Keywords: Ossicular prosthesis; Speech audiometry; Bone conduction; Audiometry; Hearing aids

\footnotetext{
Trabalho realizado na Divisão de Saúde Auditiva, Hospital de Reabilitação de Anomalias Craniofaciais, Universidade de São Paulo - USP - Bauru (SP), Brasil. ${ }^{1}$ Programa de Pós-graduação em Fonoaudiologia, Faculdade de Odontologia de Bauru - FOB, Universidade de São Paulo - USP - Bauru (SP), Brasil.

${ }^{2}$ Divisão de Saúde Auditiva, Hospital de Reabilitação de Anomalias Craniofaciais - HRAC, Universidade de São Paulo - USP - Bauru (SP), Brasil.

${ }^{3}$ Hospital de Reabilitação de Anomalias Craniofaciais - HRAC, Universidade de São Paulo - USP - Bauru (SP), Brasil.

${ }^{4}$ Curso de Medicina, Faculdade de Odontologia de Bauru - FOB, Universidade de São Paulo - USP - Bauru (SP), Brasil.

${ }^{5}$ Departamento de Fonoaudiologia, Faculdade de Odontologia de Bauru - FOB, Universidade de São Paulo - USP - Bauru (SP), Brasil.

Conflito de interesses: Não.

Contribuição dos autores: $\mathrm{BC}$ realizou a análise dos dados e elaboração do manuscrito; TSSS foi responsável pela tabulação, correções e adequações necessárias para publicação; ECB atualizou a revisão de literatura para contextualizar o trabalho; LFML supervisionou a elaboração e analisou criticamente o manuscrito; KFA supervisionou a coleta, tabulação e análise dos dados e orientou as etapas de execução e elaboração do manuscrito; RVBN foi responsável pelo delineamento do estudo e orientação geral das etapas de execução.
}

Financiamento: Nada a declarar.

Autor correspondente: Brenda Catalani. E-mail: brenda.catalani@usp.br

Recebido: Setembro 01, 2020; Aceito: Março 05, 2021 


\section{INTRODUÇÃO}

Rotineiramente, a reabilitação de indivíduos com deficiência auditiva é realizada por meio da adaptação de aparelhos de amplificação sonora individual (AASI), associada ao processo terapêutico fonoaudiológico. Contudo, em indivíduos com deficiência auditiva decorrente de malformações congênitas ou adquiridas, de orelha média, atresia/microtia congênita e otite média crônica, esses dispositivos podem não ser passíveis de $\operatorname{adaptação}^{(1)}$.

Como alternativa terapêutica para esses indivíduos, existe uma diversidade de dispositivos eletrônicos que diferem não apenas pela forma como o som é transmitido até a cóclea, mas também pelos procedimentos clínicos envolvidos em sua adaptação, que pode ser ambulatorial ou ambulatorial e cirúrgica.

No contexto ambulatorial, encontra-se o aparelho de amplificação sonora individual por condução óssea (AASI VO), composto por um arco de metal e um vibrador que, em contato com a mastoide, promove a estimulação coclear, sem que seja necessária a passagem do som através das orelhas externa e média, consecutivamente ${ }^{(2)}$. Todavia, esse tipo de AASI pode causar irritação ou dor de cabeça, uma vez que o transmissor está pressionado firmemente contra a pele da mastoide. Além disso, há relatos de algumas pessoas referindo interferência na qualidade do som e reconhecimento de fala, conforme a mudança de posição do transmissor ${ }^{(3)}$.

Para o grupo de próteses que exige o procedimento cirúrgico, existem as próteses de orelha média, com duas partes: a interna, composta pelo receptor/modulador cirurgicamente implantado e o processador de áudio externo, que é colocado sobre o couro cabeludo e permanece na sua posição por atração magnética entre as duas partes. $\mathrm{Na}$ extremidade do fio condutor, há um transdutor de flutuação de massa que pode ser inserido na bigorna, janela oval ou janela redonda ${ }^{(4)}$.

Por fim, existem as próteses auditivas ancoradas no osso (PAAO), implantadas pela primeira vez em $1977^{(5)}$ e, no Brasil, em 1997, em um hospital, em indivíduos sindrômicos com perda auditiva condutiva e mista. Inicialmente, essas próteses, idealizadas para contemplar algumas situações clínicas específicas, passaram, com a evolução tecnológica, a ter os critérios de indicação ampliados, incluindo, atualmente, indivíduos com perda auditiva sensorioneural unilateral profunda ${ }^{(6)}$.

As PAAO compreendem duas partes: uma posicionada cirurgicamente e outra, externa, que constitui o processador de som. São chamadas de percutâneas as PAAO cujo acoplamento com a unidade externa ocorre por meio de um pino de titânio implantado ao osso, e são chamadas de transcutâneas as próteses que o acoplamento com a unidade externa ocorre por meio de um imã. As PAAO que vibram o osso através da pele (skin drive) são chamadas de transcutâneas passivas, com imãs implantados sob a pele, disponíveis comercialmente como Baha ${ }^{\circledR}$ Attract e Sophono $^{\circledR}$. As PAAO que estimulam diretamente o osso (direct drive) são chamadas de percutâneas (Baha ${ }^{\circledR}$ Connect e Ponto ${ }^{\circledR}$ ) e transcutâneas ativas (Bonebridge ${ }^{\circledR}$ e Osia) ${ }^{(7)}$.

Concomitante ao avanço das alternativas terapêuticas, há uma constante preocupação das empresas para que as novas tecnologias proporcionem a melhora do processamento do sinal sonoro recebido pelo usuário das $\mathrm{PAAO}$, com consequente benefício na percepção de fala e maior conforto em ambiente ruidoso ${ }^{(8)}$. Nessa perspectiva, a empresa Oticon Medical desenvolveu e lançou, em 2009, o Sistema Ponto ${ }^{\circledR}$, uma PAAO percutânea que disponibiliza aspectos avançados do processamento do sinal, tais como direcionalidade adaptativa, supressão do ruído de vento, redução de ruído ambiental, entre outros, recursos também presentes nos aparelhos de amplificação sonora por condução aérea ${ }^{(9)}$.

Recentes pesquisas foram realizadas com o Sistema Ponto ${ }^{\circledR}$, comparando diferentes modelos e potências ${ }^{(10)}$, a estabilidade do implante ${ }^{(11)}$, ou, até mesmo, avaliando a discriminação auditiva após o uso desse dispositivo ${ }^{(12)}$, porém, em âmbito nacional, não há registros do benefício obtido pelo indivíduo com deficiência auditiva após a cirurgia para PAAO com o Sistema Ponto ${ }^{\circledR}$.

Desse modo, esta pesquisa teve por objetivo descrever os benefícios nos limiares auditivos e no desempenho de reconhecimento de sentenças no silêncio e no ruído em indivíduos com deficiência auditiva condutiva ou mista, nas condições pré e pós-cirúrgicas com a PAAO Sistema Ponto ${ }^{\circledR}$.

\section{MÉTODOS}

Trata-se de um estudo com delineamento primário, observacional e retrospectivo, de seguimento longitudinal, aprovado no Comitê de Ética em Pesquisa do Hospital de Reabilitação de Anomalias Craniofaciais (HRAC/USP), sob parecer 3.417.010. Por se tratar de um estudo retrospectivo por meio de coleta de dados já presentes nos prontuários, não sendo necessárias informações adicionais ou divulgação de imagens dos pacientes, o Termo de Consentimento Livre e Esclarecido (TCLE) foi dispensado.

A coleta de dados foi realizada por meio da análise documental de prontuários de pacientes matriculados na Divisão de Saúde Auditiva do Hospital de Reabilitação de Anomalias Craniofaciais da Universidade de São Paulo - HRAC/USP. O programa possui protocolo clínico padronizado para a avaliação e o acompanhamento de candidatos e usuários de PAAO. Os dados secundários foram coletados por meio do sistema de software de gestão hospitalar Tasy, no período de julho a agosto de 2019.

Os critérios de elegibilidade foram indivíduos com deficiência auditiva condutiva ou mista bilateral, que realizaram cirurgia da PAAO Sistema Ponto ${ }^{\circledR}$ no período, e que apresentavam os seguintes dados no prontuário: audiometria tonal liminar por conduções aérea e óssea nas condições pré e pós-cirúrgicas, sendo considerada a avaliação realizada imediatamente anterior à cirurgia; limiares na audiometria tonal em campo livre e reconhecimento de sentenças no silêncio e no ruído nas seguintes condições:

- Pré-cirúrgica (considerada a última avaliação realizada próxima à cirurgia): sem dispositivo eletrônico; com AASI por condução aérea ou óssea; com o processador Ponto Pro ${ }^{\circledR}$ acoplado a uma banda elástica;

- Pós-cirúrgica: na ativação da PAAO Sistema Ponto ${ }^{\circledR}$, que ocorre 12 semanas após a cirurgia; seis meses de uso da PAAO Sistema Ponto ${ }^{\circledR}$.

Foi considerada como critério de exclusão a falta de algum dado dentre aqueles requeridos, sendo, assim, excluídos os prontuários que não apresentaram as informações suficientes para o estudo em questão.

Os dados coletados de acordo com o procedimento aplicado estão descritos a seguir:

- Audiometria tonal liminar: limiares tonais por condução aérea obtidos nas frequências de 0,25 a $8 \mathrm{kHz}$ e limiares por condução óssea obtidos nas frequências de 0,5 a 4 kHz 
para o estímulo tom puro, apresentado por meio do fone supra-aural TDH49 - Telephonics e vibrador ósseo B71 - Radioear, respectivamente;

- Audiometria tonal em campo livre: limiares tonais obtidos nas frequências de 0,$5 ; 1 ; 2 ; 3$ e $4 \mathrm{kHz}$ para o estímulo tom modulado (Warble) com a caixa de som posicionada a $0^{\circ}$ azimute a um metro do indivíduo, em cabina acústica. Para a realização da audiometria tonal liminar e em campo livre foi utilizado o audiômetro Astera 2 Madsen - Otometrics;

- Reconhecimento de sentenças no silêncio e no ruído: as seis listas de 10 sentenças propostas por Costa et al. ${ }^{(13)}$ foram gravadas e apresentadas no silêncio e no ruído, por meio do mesmo audiômetro Astera 2 Madsen Otometrics, conectado a um amplificador em campo livre e a uma caixa acústica posicionada $0^{\circ}$ azimute, a um metro do indivíduo, em sala tratada acusticamente.

O limiar de reconhecimento de fala foi pesquisado nas condições sem e com o AASI e o Sistema Ponto ${ }^{\circledR}$, no silêncio e no ruído. Para tanto, foi utilizada a técnica ascendente-descendente (estratégia proposta por Levitt e Rabiner ${ }^{(14)}$, denominada sequência adaptativa), para a apresentação das dez sentenças. Assim, a primeira sentença foi apresentada na intensidade de 65 dBNA (decibel nível de audição) e, frente à repetição correta pelo indivíduo, a intensidade foi sendo diminuída em passos de 4 dBNA, até a ocorrência de um erro cometido pelo indivíduo, na repetição. A partir dessa intensidade, utilizaram-se passos de 2 dBNA na intensidade, ou seja, aumentaram-se 2 dBNA na apresentação da sentença em caso de erro, ou diminuíram-se 2 dBNA em caso de acerto, até finalizar a lista utilizada. Para o cálculo do limiar de reconhecimento de fala, considerou-se a intensidade em que foi obtido o primeiro erro, somando-a com as intensidades de apresentação das sentenças subsequentes. $\mathrm{O}$ valor obtido foi dividido pelo total de sentenças utilizadas na soma, estabelecendo-se, assim, o limiar de reconhecimento das sentenças no silêncio.

Em seguida, utilizou-se a mesma técnica, porém, com a apresentação do ruído competitivo na intensidade de 60 dBNA, ou seja, uma relação sinal/ruído inicial de $+5 \mathrm{~dB}$ (decibel), para a determinação do limiar de reconhecimento das sentenças no ruído. A relação sinal/ruído foi determinada pela subtração do limiar de reconhecimento de sentenças no ruído da intensidade fixa do ruído de 60 dBNA.

\section{Análise dos resultados}

Os resultados foram apresentados em forma de tabelas e gráficos. Inicialmente, os dados foram submetidos à análise estatística descritiva, sendo determinado o percentual de ocorrência para cada variável qualitativa nominal e determinados os valores de média, DP (desvio padrão), mediana, percentis 25 e 75.

Para a estatística inferencial, de acordo com o Teste de Kolmogorov-Smirnov, os limiares auditivos obtidos na audiometria tonal por conduções aérea e óssea e em campo livre não apresentaram distribuição normal ( $\mathrm{p} \geq 0,05)$, o que determinou o uso de testes não paramétricos. Para comparar os limiares tonais por conduções aérea e óssea obtidos sem dispositivo e aqueles nas diferentes condições, utilizou-se o Teste Wilcoxon, enquanto que, para aqueles obtidos em campo livre, foram aplicados o Teste de Friedman e, posteriormente, o Teste Tukey. Já a análise dos dados referentes ao reconhecimento de sentenças no silêncio e no ruído, constatou distribuição normal, sendo, assim, utilizado o teste paramétrico Variância com Medidas Repetidas e, posteriormente, Teste Tukey. Para as análises inferenciais, foi fixado nível de significância de 0,05 . O software de análise estatística utilizado foi o SPSS, versão 21.

\section{RESULTADOS}

Diante dos critérios previamente estabelecidos, foram analisados os prontuários de 10 indivíduos, sendo 5 do gênero masculino e 5 do gênero feminino, com média de idade de 27 anos, mínima de 15 anos e máxima de 49 anos, adaptados unilateralmente com o Sistema Ponto Pro ${ }^{\circledR}$. Desses indivíduos, 6 eram portadores de malformação de orelha bilateral e 4 apresentavam sequelas de otite média crônica bilateral e utilizavam AASI por condução aérea ou óssea, anterior à cirurgia. A caracterização da casuística está apresentada no Quadro 1.

Quadro 1. Caracterização da casuística

\begin{tabular}{|c|c|c|c|c|}
\hline $\mathbf{n}$ & Gênero & Idade da ativação (anos) & Tipo e grau de perda auditiva & Lado implantado \\
\hline \multirow[t]{2}{*}{1} & $\mathrm{~F}$ & 32 & OD: condutiva severa & $\mathrm{D}$ \\
\hline & & & OE: condutiva moderada & \\
\hline 2 & M & 27 & Bilateral: mista severa & $E$ \\
\hline \multirow[t]{2}{*}{3} & $\mathrm{~F}$ & 25 & OD: condutiva moderada & $E$ \\
\hline & & & OE: condutiva severa & \\
\hline 4 & M & 21 & Bilateral: condutiva severa & $E$ \\
\hline \multirow[t]{2}{*}{5} & M & 30 & OD: condutiva moderada & D \\
\hline & & & OE: mista moderada & \\
\hline 6 & $\mathrm{~F}$ & 24 & Bilateral: condutiva moderada & $\mathrm{D}$ \\
\hline 7 & $\mathrm{~F}$ & 15 & Bilateral: mista moderada & $\mathrm{D}$ \\
\hline \multirow[t]{2}{*}{8} & M & 49 & OD: mista profunda & $\mathrm{D}$ \\
\hline & & & OE: mista moderada & \\
\hline \multirow[t]{2}{*}{9} & M & 27 & OD: sensorioneural profunda & $E$ \\
\hline & & & OE: mista moderada & \\
\hline \multirow[t]{2}{*}{10} & $\mathrm{~F}$ & 20 & OD: mista moderada & $\mathrm{D}$ \\
\hline & & & OE: sensorioneural profunda & \\
\hline
\end{tabular}

Legenda: $\mathrm{n}$ = sujeito número; $\mathrm{F}=$ feminino; $\mathrm{M}=$ masculino; $\mathrm{OD}=$ orelha direita; $\mathrm{OE}=$ orelha esquerda; $\mathrm{D}=$ direito; $\mathrm{E}=\mathrm{esquerdo}$ 
Os limiares da audiometria tonal liminar por condução aérea e óssea para as frequências testadas nas etapas pré-cirúrgica e pós-cirúrgica, com os indivíduos sem dispositivos eletrônicos, não apresentaram diferenças significantes $(\mathrm{p}>0,05)$ (Figura 1).

Em relação aos limiares tonais da audiometria em campo livre nas três condições pré-cirúrgicas (sem dispositivo eletrônico; com AASI por condução aérea ou óssea e com o processador Ponto Pro ${ }^{\circledR}$ acoplado a uma banda elástica) e as duas condições pós-cirúrgicas (ativação da PAAO Sistema Ponto ${ }^{\circledR}$ e 6 meses após a ativação), foi possível constatar melhora significativa dos limiares, ao comparar aqueles encontrados na audiometria em campo livre sem uso do dispositivo eletrônico com as demais condições de avaliação, exceto para a condição do indivíduo com AASI, na qual a diferença ocorreu apenas para as frequências de 2, 3 e $4 \mathrm{kHz}$. Não houve diferença significativa entre os limiares obtidos com o indivíduo utilizando o Sistema Ponto Pro $^{\circledR}$ acoplado a uma banda elástica (momento pré-cirúrgico) e nos momentos pós-cirúrgicos (Figura 2).

Quanto ao desempenho no reconhecimento de sentenças no silêncio, foram comparadas as mesmas condições pré e pós-cirúrgicas citadas anteriormente, ocorrendo diferença entre os resultados, quando comparados àqueles encontrados na

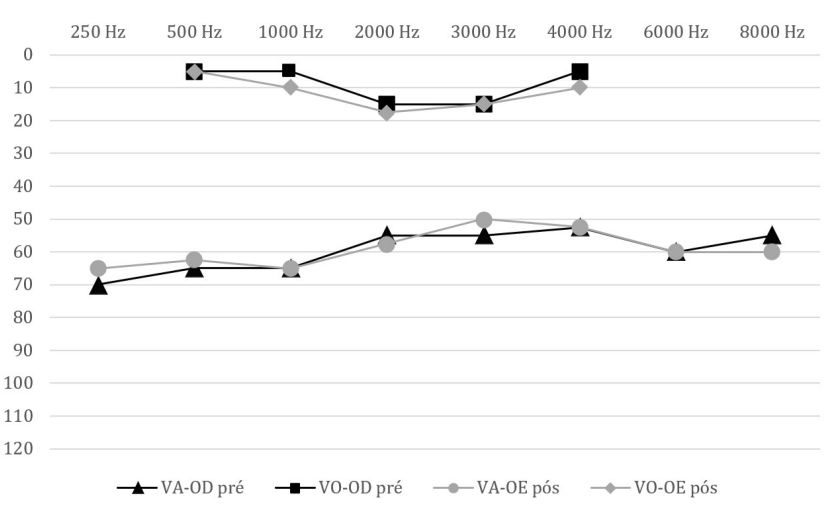

Figura 1. Mediana dos limiares tonais (dBNA) obtidas na audiometria tonal liminar, por conduções aérea e óssea, nas condições pré e pós-cirúrgicas

Legenda: $\mathrm{HZ}=$ hertz; $\mathrm{VA}-\mathrm{OD}$ = via aérea-orelha direita; $\mathrm{VO}-\mathrm{OD}$ = via óssea-orelha direita; $\mathrm{VA}-\mathrm{OE}=$ via aérea-orelha esquerda; $\mathrm{VO}-\mathrm{OE}$ = via óssea-orelha esquerda; Teste Wilcoxon $p>0,05$

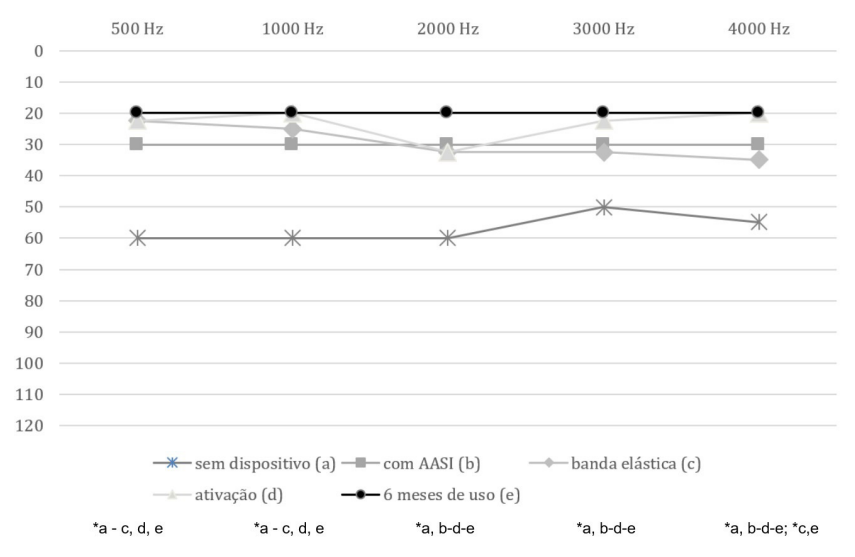

Figura 2. Mediana dos limiares tonais (dBNA) obtidos na audiometria em campo livre, nas condições pré e pós-cirúrgicas

Legenda: $\mathrm{Hz}=$ hertz; $\mathrm{AASI}=$ aparelho de amplificação sonora individual; Teste Friedman ( $p<0,001)$; Letras diferentes $(a, b, c, d, e)$ na linha indicam diferenças significativas $(p \leq 0,05)$ entre as condições avaliadas (Teste Tukey) audiometria em campo livre sem uso do dispositivo eletrônico e a todas as demais condições de avaliação nas quais o indivíduo estava usando o AASI ou Ponto Pro ${ }^{\circledR}$ (pré e pós-cirúrgico). Não houve diferença significativa entre os limiares obtidos com o indivíduo utilizando o Sistema Ponto acoplado a uma banda elástica (pré-cirúrgico) e as condições de avaliação pós-cirúrgica (Tabela 1).

Nos dados referentes ao desempenho no reconhecimento de sentenças no ruído, também comparados entre todas as condições pré e pós-cirúrgicas já descritas, encontrou-se diferença entre a condição sem dispositivo eletrônico, quando comparada às condições com o processador Ponto Pro ${ }^{\circledR}$ acoplado a uma banda elástica, na ativação e 6 meses após a ativação, mas não com o indivíduo usando o AASI. Não foram observadas diferenças entre as demais condições de teste (Figura 3).

\section{DISCUSSÃO}

Por décadas, o fonoaudiólogo, na prática clínica, ao se deparar com um indivíduo com malformação de orelha externa e/ou média unilateral ou bilateral, com consequente perda auditiva condutiva, sentia sua atuação terapêutica restrita, visto a escassez de opções tecnológicas para a estimulação auditiva. O AASI por condução óssea era a possibilidade mais acessível, não por ser de fácil adaptação e aceitação, mas por não requerer o ato cirúrgico. A literatura da área descreve os

Tabela 1. Limiar de reconhecimento de sentenças no silêncio e relação sinal/ruído, de acordo com as condições de avaliação

\begin{tabular}{lccccc}
\hline \multirow{2}{*}{ Condições } & \multicolumn{2}{c}{ Silêncio (dBNA) } & \multicolumn{3}{c}{ Relação sinal/ruído (dB) } \\
\cline { 2 - 3 } \cline { 5 - 6 } & Média & DP & & Média & DP \\
\hline Sem & $56,8^{\mathrm{a}}$ & 5,6 & & $3,6^{\mathrm{a}}$ & 1,7 \\
dispositivo & & & & $0,5^{\mathrm{b}}$ & 3,5 \\
Com AASI & $31,4^{\mathrm{b}, \mathrm{a}}$ & 8,3 & & $-1,4^{\mathrm{c}, \mathrm{a}}$ & 3,7 \\
$\begin{array}{l}\text { Com banda } \\
\text { elástica }\end{array}$ & $33,2^{\mathrm{c}, \mathrm{a}}$ & 9,2 & & \\
Ativação & $29,5^{\mathrm{d}, \mathrm{a}}$ & 11,4 & & $-2,6^{\mathrm{d}, \mathrm{a}}$ & 2,9 \\
$\begin{array}{l}\text { Seis meses } \\
\text { de uso }\end{array}$ & $25,9^{\mathrm{e}, \mathrm{a}}$ & 8,9 & & $-3,4^{\mathrm{e}, \mathrm{a}}$ & 3,6 \\
\hline
\end{tabular}

Teste Variância com Medidas Repetidas. Letras diferentes (a,b,c,c,e,e) na linha indicam diferenças significativas $(p \leq 0,05)$ entre as condições avaliadas (Teste Tukey) Legenda: $\mathrm{dBNA}=$ decibel nível de audição; $\mathrm{dB}=$ decibel; $\mathrm{DP}=$ desvio padrão; AASI = aparelho de amplificação sonora individual

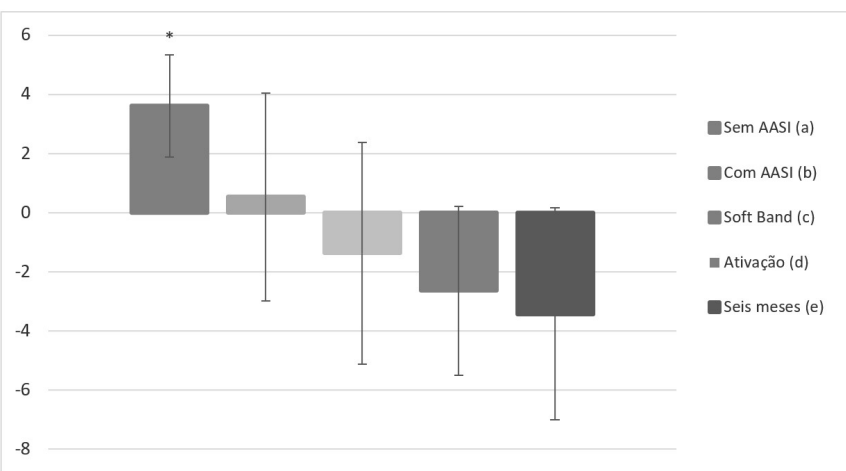

Figura 3. Relação sinal/ruído (dB) obtida, de acordo com as condições de avaliação

Legenda: AASI = aparelho de amplificação sonora individual; *a - c,d,e; Letras diferentes $(a, b, c, d, e)$ na coluna indicam diferenças significativas $(p \leq 0,05)$ entre as condições avaliadas 
aspectos desfavoráveis desse dispositivo eletrônico, que vão desde sensações desconfortantes ao usuário à dificuldade de mantê-lo na posição correta para alcançar a estimulação adequada e de qualidade, principalmente em crianças.

Na perspectiva do indivíduo e/ou família, como a perda auditiva decorrente é comumente condutiva, de grau leve ou moderado e, a princípio, com pouco impacto no desenvolvimento da linguagem e comunicação oral, a adesão do indivíduo e/ ou família ao tratamento geralmente não era efetiva, pois o interesse, na maioria das vezes, era pela cirurgia plástica para a correção anatômica, sustentado, principalmente, pelo receio dos preconceitos observados no convívio social.

Contudo, o maior conhecimento sobre o impacto da perda auditiva condutiva no processamento auditivo, com possíveis dificuldades acadêmicas, associado aos avanços na tecnologia dos aparelhos auditivos por condução óssea, tem-se observado, nos últimos anos, maior interesse dos profissionais pelas pesquisas com usuários de PAAO, com o intuito de sustentar a prática clínica.

Nesse contexto, o presente estudo analisou os benefícios obtidos da indicação unilateral do Sistema Ponto Pro ${ }^{\circledR} \mathrm{em}$ indivíduos com perda auditiva bilateral (Quadro 1).

O Sistema Ponto ${ }^{\circledR}$ é uma PAAO, ou seja, um dispositivo capaz de produzir sensação sonora, independente da função da orelha externa e média ${ }^{(15,16)}$, pois transmite a energia por meio da vibração do crânio. Trata-se de um dispositivo implantável percutâneo, ou seja, o acoplamento com a unidade externa ocorre por meio de um pino de titânio implantado no osso da mastoide.

O procedimento cirúrgico não é considerado complexo pelo profissional especialista, pois as estruturas da orelha não são manipuladas durante a intervenção. Assim, não é esperada modificação nos limiares obtidos na audiometria tonal por conduções aérea e óssea após a cirurgia, como foi observado no presente estudo (Figura 1) e condizente com o descrito anteriomente ${ }^{(12)}$.

Adicionalmente, estudos com diferentes próteses percutâneas já demonstraram sua efetividade, com melhora significativa dos limiares tonais em todas as frequências na audiometria em campo livre ${ }^{(15-18)}$, quando comparadas as condições sem e com o dispositivo eletrônico. No entanto, o maior ganho tem sido descrito para as frequências mais altas ${ }^{(19)}$ ou médias ${ }^{(20)}$, o que diverge dos resultados obtidos neste estudo, que manteve um ganho similar entre todas as frequências pesquisadas, 0,5 a $4 \mathrm{kHz}$ (Figura 2).

A percepção de fala foi analisada em estudos anteriores por meio do reconhecimento de palavras monossílabas e dissílabas, com melhora significativa no momento pós-adaptação do dispositivo, tanto no silêncio ${ }^{(15,17,19,21)}$ quanto no silêncio e no ruído ${ }^{(21)}$. Semelhante achado foi obtido na percepção de fala de sentenças no silêncio (Tabela 1).

Cabe destacar, contudo, que foi constatado que o desempenho na percepção de fala no silêncio, no momento da ativação e após seis meses de uso do Sistema Ponto ${ }^{\circledR}$, não demonstrou diferença significativa (Tabela 1), o que sugere que o benefício obtido pela amplificação por condução óssea é imediato nos indivíduos usuários de AASI por condução aérea ou óssea anterior à cirurgia. Estudos futuros devem ser realizados para verificar se esse resultado é mantido, mesmo em indivíduos que não são usuários prévios de AASI, seja por condução aérea, ou óssea.

Para a percepção de fala com ruído competitivo, verificou-se melhor desempenho na situação de escuta difícil nas condições com o uso do Sistema Ponto ${ }^{\circledR}$, resultado não observado para o
AASI por condução aérea ou óssea (Tabela 1), achado condizente com estudos prévios ${ }^{(22,23)}$.

Contrário ao descrito anteriormente ${ }^{(12)}$, o benefício do Sistema Ponto $^{\circledR}$ para a percepção da fala no ruído foi observado, mesmo sendo uma adaptação unilateral, achado que permite questionar o quanto a estimulação por condução óssea propicia, também, a estimulação da cóclea contralateral, o que simularia a estimulação bilateral, aspecto que deve ser explorado em estudos futuros.

Assim como na habilidade auditiva de detecção, não houve diferença significativa no desempenho para percepção de fala no ruído na ativação e após seis meses, apesar da média da relação sinal/ruído mais negativa após seis mês (Figura 3), resultado semelhante ao de um estudo com um ano de uso do dispositivo (24).

De modo geral, os resultados obtidos com o processador Ponto Pro $^{\circledR}$ acoplado a uma banda elástica não diferiram significativamente dos resultados pós-cirúrgicos (ativação e seis meses de uso), tanto na audiometria tonal em campo livre, quanto na percepção de fala no silêncio e no ruído. Sendo assim, esse dispositivo possibilita a decisão mais consciente do indivíduo em relação à cirurgia, com expectativas reais no tocante aos benefícios pós-cirúrgicos.

É importante ressaltar que, apesar das limitações do presente estudo quanto ao número de participantes e heterogeneidade da casuística, é possível afirmar que o uso de PAAO percutânea promove melhora significativa nos dados audiológicos e reconhecimento de fala do usuário.

\section{CONCLUSÃO}

O Sistema Ponto ${ }^{\circledR}$ propiciou benefício nas habilidades auditivas de detecção em todas as frequências testadas, demonstrado pelos limiares obtidos em campo livre, como também no reconhecimento de sentenças no silêncio e no ruído.

\section{REFERÊNCIAS}

1. Snik FM, Mylanus EAM, Cremers WRJ. The bone-anchored hearing aid: a solution for previously unresolved otologic problems. Otolaryngol Clin North Am. 2001;34(2):365-72. http://dx.doi.org/10.1016/S00306665(05)70336-4. PMid:11382575.

2. Paccola ECM. Aparelho de amplificação sonora individual por condução óssea e malformações congênitas das orelhas: caracterização e análise do benefício e satisfação [dissertação]. Bauru: Hospital de Anomalias Craniofaciais, Universidade de São Paulo; 2007.

3. Mylanus EA, Snik AF, Jorritsma FF, Cremers CW. Audiologic results for the bone-anchored hearing aid HC220. Ear Hear. 1994;15(1):87-92. http://dx.doi.org/10.1097/00003446-199402000-00010. PMid:8194683.

4. Bittencourt AG, Burke PR, Jardim IDS, Brito R, Tsuji RK, Fonseca ACO, Bento RF. Implantable and semi-Implantable hearing aids: a review of history, indications and surgery. Int Arch Otorhinolaryngology. 2014;18(3):303-10.

5. Tjellström A, Lindström J, Hallén O, Albrektsson T, Brånemark PI. Direct bone anchorage of external hearing aids. J Biomed Eng. 1983;5(1):59-63. http://dx.doi.org/10.1016/0141-5425(83)90080-8. PMid:6827820. 
6. Dumper J, Hodgetts B, Liu R, Brandner N. Indications for boneanchored hearing AIDS: a functional outcome study. J Otolaryngol Head Neck Surg. 2009;38(1):96-105. PMid:19344618.

7. Reinfeldt S, Håkansson B, Taghavi H, Eeg-Olofsson M. New developments in bone-conduction hearing implants: a review. Med Devices. 2015;8:79-93. http://dx.doi.org/10.2147/MDER.S39691. PMid:25653565.

8. Gawęcki W, Stieler OM, BalcerowiakA, Komar D, Gibasiewicz R, Karlik $\mathrm{M}$, et al. Surgical, functional and audiological evaluation of new Baha Attract system implantations. Eur Arch Otorhinolaryngol. 2016;273:312330. http://dx.doi.org/10.1007/s00405-016-3917-5. PMid:26899281.

9. Oticon Medical. The Ponto bone-anchored hearing system from Oticon Medical [product catalog]. Askim: Oticon Medical; 2009.

10. Bianchi F, Wendt D, Wassard C, Maas P, Lunner T, Rosenbom $\mathrm{T}$, et al. Benefit of higher maximum force output on listening effort in bone-anchored hearing system users: a pupillometry study. Ear Hear. 2019;40(5):1220-32. http://dx.doi.org/10.1097/AUD.0000000000000699. PMid:30807542.

11. Westover L, Faulkner G, Hodgetts W, Raboud D. Comparison of implant stability measurement devices for bone-anchored hearing aid systems. J Prosthet Dent. 2018;119(1):178-84. http://dx.doi. org/10.1016/j.prosdent.2017.02.021. PMid:28533009.

12. Celikgun B, Kalcioglu MT. Assessment of discrimination ability in ipsilateral and contralateral ears with a unilateral bone-anchored hearing system. Ear Nose Throat J. 2017;96(8):297-310. http://dx.doi. org/10.1177/014556131709600816. PMid:28846784.

13. Costa MJ, Iório MCM, Mangabeira-Albernaz PL. Reconhecimento de fala: desenvolvimento de uma lista de sentenças em português. Acta Awho. 1997;16(4):164-73.

14. Levitt H, Rabiner LR. Binaural release from masking for speech and gain in intelligibility. J Acoust Soc Am. 1967;42(3):601-8. http:// dx.doi.org/10.1121/1.1910629. PMid:6073973.

15. Boleas-Aguirre MS, Plano MDB, Erenchun Lasa IR, Beroiz BI. Audiological and subjective benefit results in bone-anchored hearing device users. Otol Neurotol. 2012;33(4):494-503. http://dx.doi. org/10.1097/MAO.0b013e31824b76f1. PMid:22472615.
16. Bahmad F Jr, Cardoso CC, Caldas FF, Barreto MADSC, Hilgenberg AMDS, Teixeira MS, et al. Hearing rehabilitation through bone-conducted sound stimulation: preliminary results. Int Arch Otorhinolaryngol. 2019;23(01):12-7. http://dx.doi.org/10.1055/s-0038-1670694. PMid:30647778.

17. Caruso A, Giannuzzi AL, Sozzi V, Sanna M. Bone anchored hearing implants without skin thinning: the Gruppo Otologico surgical and audiological experience. Eur Arch Otorhinolaryngol. 2017;274(2):695700. http://dx.doi.org/10.1007/s00405-016-4305-x. PMid:27637751.

18. Милешина НА, Осипенков СС, Таварткиладзе ГА. Алгоритм ведения больных с врожденными пороками развития наружного и среднего уха. Vestn Otorinolaringol. 2013;83(4):51-5.

19. Flynn MC, Hedin A, Halvarsson G, Good T, Sadeghi A. Hearing performance benefits of a programmable power baha ${ }^{\circledR}$ sound processor with a directional microphone for patients with a mixed hearing loss. Clin Exp Otorhinolaryngol. 2012;5(Suppl. 1):S76-81. http://dx.doi. org/10.3342/ceo.2012.5.S1.S76. PMid:22701154.

20. Spitzer JB, Ghossaini SN, Wazen JJ. Evolving applications in the use of bone-anchored hearing aids. Am J Audiol. 2002;11(2):96-103. http://dx.doi.org/10.1044/1059-0889(2002/011). PMid:12691219.

21. Lagerkvist H, Carvalho K, Holmberg M, Petersson U, Cremers C, Hultcrantz M. Ten years of experience with the Ponto bone anchored hearing system-a systematic literature review. Clin Otolaryngol. 2020;45(5):667-80. http://dx.doi.org/10.1111/coa.13556. PMid:32386454.

22. Carlsson P, Håkansson B, Rosenhall U, Tjellström A. A speech-tonoise ratio test with the bone-anchored hearing aid: a comparative study. Otolaryngol Head Neck Surg. 1986;94(4):421-6. http://dx.doi. org/10.1177/019459988609400402. PMid:3086800.

23. Wazen JJ, Van Ess MJ, Alameda J, Ortega C, Modisett M, Pinsky K. The Baha system in patients with single-sided deafness and contralateral hearing loss. Otolaryngol Head Neck Surg. 2010;142(4):554-9. http:// dx.doi.org/10.1016/j.otohns.2009.12.047. PMid:20304277.

24. Saliba I, Woods O, Caron C. BAHA results in children at one year followup: a prospective longitudinal study. Int J Pediatr Otorhinolaryngol. 2010;74(9):1058-62. http://dx.doi.org/10.1016/j.ijporl.2010.06.004. PMid:20594601. 